\title{
Optimized Design Method of the Prefabricated Hole for Flanging Forming of Square Tributary Junction on Spherical Shell
}

\author{
Zhong Lei WANG ${ }^{1, a}$, Shi Ke HAO ${ }^{1}$, Gang CHENG ${ }^{1}$, Jin Jing XU ${ }^{1}$ \\ ${ }^{1}$ School of Mechanical and Electrical Engineering, Shandong Jianzhu University, Jinan 250101, China.
}

\begin{abstract}
The key problem for flanging forming of square tributary junction on spherical shell is to determine the shape and sizes of prefabricated hole. In order to investigate precise shape and sizes of the prefabricated hole, an optimized design model was established. The flanging forming process was simulated using the software of FE-simulation and the target function for optimized design was calculated based on results of the simulations. In order to increase the convergence rate of the optimized design, a mathematical model of prefabricated hole for flanging forming on spherical shell was established, the results were used as initial values for optimized design. To verify its accuracy, the optimized design was carried out on a typical flanging forming of square tributary junction on spherical shell. The results of the optimized design meet the technological requirements.
\end{abstract}

\section{Introduction}

With rapid development of economy and technology, especially the rapid development of urban pipe network, petrochemical industry, water conservancy project, oil and gas transportation, the large-scale pipe network is rapidly developed.The demands for for pipe joins, which is key component for connecting of large tube nets, is also increasing. For the process design of flanging of square tributary junction on spherical shell, the critical issue is to determine the shape and sizes of pre-machined hole precisely, for this an optimized design method is researched in this paper.

Luo Ya Tao[1]simplified the pre-machined hold as ellipse, and calculated the long and short axis of the ellipse through special positions, and further investigated the shape and sizes of the pre-machined ellipse.Liu Jian Shen et. [2-4] carried out experimental and numerical study on flanging on thick wall thickness tube. In order to obtain better quality part of the flanging forming, Chen Y.L[5] compared the two specific cases of the flanging forming process by using finite element software DEFORM.Gu Li[6]simplified the pre-machined hole as ellipse to optimize the parameters for flanging of T-shape tube with greater diameter, in which the long and short axis of the ellipse as optimized design variables, the neural network system as the optimized target function solver, and the genetic algorithm is used.

In the aforementioned researches the optimized design method based on optimized algorithm is of high degree of automation and reliability. Yet the accuracy of the optimized design method to determination of prefabricated hole is not high, the reason is that the prefabricated hole by simplifying is regarded as an ellipse, it could not describe the shape of the prefabricated hole precisely. In this paper an optimized model is established, in which the shape of the prefabricated hole is controlled with spline curves, and spline curves are controlled with points, so a high accuracy of prefabricated hole in the optimized design can be achieved.

\section{Establishment of Optimized Model}

Optimization model including three aspects: the optimal objective function, optimization design variables and constraint conditions. Optimization problem of prefabricated hole of flanging forming on spherical shell can be described that choosing the size of appropriate prefabricated hole make the height of the flanging port meets the requirements of high value. Goal of optimization design is to make the height of the flanging port close to ideal flanging height, so we can put the minimum of the geometric average of the difference of the flanging port number of discrete point height and ideal height as the optimization objective function. Optimal design variables of the optimization model is the geometry of prefabricated hole of flanging forming. Since the prefabricated hole is a continuous space geometry curve, however the optimal design variables are limited data, so the continuous space geometry curve must be discrete. Some key nodes are used as the design variables of optimization design and the prefabricated hole is simplified as a spline curve which is determined by the key nodes. The prefabricated hole is a space curve, so the key points should be described by the three-dimensional 
coordinates. However, the prefabricated hole is on the spherical shell, so the $\mathrm{Z}$ coordinates can be calculated when its $(X, Y)$ coordinates can be determined. From what has been discussed above, the optimal design variables is the $(\mathrm{X}, \mathrm{Y})$ coordinates of the key points of the hole.

In order to ensure the performance of the flanging process successfully and the parts of flanging forming can meet the technological requirements, the optimization process must meet the requirements of the flanging process. So it is the constraint conditions of the optimization model that there aren't defects, such as wrinkling and cracking, on the flanging forming workpiece. In order to meet the requirements of the following welding technology, the roughness of port must be guaranteed. So the maximum height difference of the port will be used as another constraint conditions of optimization model.

In summary, the optimization model is described as follow:

Design variables of the optimization model: $\left(x_{1}, y_{1}\right),\left(x_{2}, y_{2}\right) \Lambda\left(x_{n}, y_{n}\right)$

Target function of the optimization model:

$$
\begin{aligned}
& F\left(x_{1}, y_{1}, x_{2}, y_{2} \Lambda x_{n}, y_{n}\right)= \\
& \sqrt{\left(\left(H_{1}-H\right)^{2}+\left(H_{2}-H\right)^{2}+\Lambda+\left(H_{n}-H\right)^{2}\right) / n}
\end{aligned}
$$

Constraint conditions of the optimization model:

(1) cracks or wrinkles should not be aroused on the parts.

(2) $H_{\max }-H_{\min } \leq \Delta H$.

In which, $\left(x_{1}, y_{1}\right),\left(x_{2}, y_{2}\right) \Lambda\left(x_{n}, y_{n}\right)$ are coordinates of discrete points of the prefabricated hole, $\left(\begin{array}{llll}H_{1} & H_{2} & \Lambda & H_{n}\end{array}\right)$ are flanged height in various positions, $H$ is the ideal height of workpiece, $H_{\text {max }}, H_{\text {min }}$ are maximal and minimal height of flanged in all positions; $\Delta H$ is maximal allowed height tolerance, in this paper $\Delta H=0.25 \mathrm{~mm}$.

\section{Initial Values of Optimized Variables}

The convergence and convergence rate in optimized design are closely related with initial value of design variables, so an important work for optimized design is to ascertain the initial values for the optimized design variables. In order to determine the initial value of optimization variables, the mathematical model of prefabricated hole is established. In flange-forming process, the flowing in the thickness of the sheet in the forming region is more obvious than that in the radial direction. So the method of equal length is usually used to calculate the prefabricated hole of flange-forming.

In order to study the problem, the main coordinate system is set up. The axis of the spherical shell is the $Z$ axis of coordinate system and the center of the spherical shell is the origin of coordinates. The straight lines which parallel to the flange square port side and through the origin line respectively is the $\mathrm{X}$ and $\mathrm{Y}$ axis. It assumes that the length of flanging hole sides are $\mathrm{L}$ and $\mathrm{W}$ and the radius of the round corners in the neutral layer is $\mathrm{R} 3$. It assumes that spherical shell radius in neutral layer radius is $R 1$,flange corner in neutral layer radius is $\mathrm{R} 2$ and sheet thickness is $t$. If the forming part is cut down by the plane through the $\mathrm{Z}$ axis, we could get the schematic section as shown in figure 1.

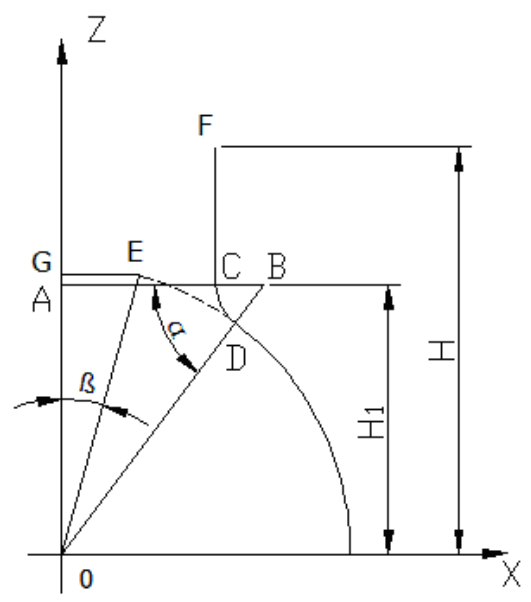

Figure 1. Schematic section of flanging forming workpiece.

From Figure1, we can see that $\mathrm{H} 1$ can be obtained by Eq2 using Pythagorean theorem.

$$
H_{1}=\sqrt{(\overline{O D}+\overline{D B})^{2}-(\overline{A C}+\overline{C B})^{2}}
$$

In which, $\overline{C B}=\overline{B D}=R_{2}, \overline{O D}=R_{1}$.

The geometrical relationship can be obtained from the Figure1.

$$
\begin{gathered}
\overline{C F}=H-H_{1} \\
\tilde{C D}=R_{2} \arccos \frac{\overline{A C}+R_{2}}{R_{1}+R_{2}}
\end{gathered}
$$

As shown is figure 1,the length of material before forming is the length of arc $\tilde{D E}$ and the length of material after forming is the sum of length of arc $\tilde{C D}$ and length of segment $\overline{C F}$.According to the method of equal length, Eq5 can be known.

$$
\begin{aligned}
& \tilde{D E}=\overline{C F}+\tilde{C D} \\
& \beta=\pi / 2-\tilde{D E} / R_{1}-\alpha \\
& \overline{E G}=R_{2} \sin \beta
\end{aligned}
$$

Obtained line segment $\overline{E G}$, the coordinates of point $\mathrm{E}$ in main coordinate system can be obtained.In order to calculate the length of segment $\overline{A C}$, we analyze the view of flanging forming workpiece in $X O Y$ plane, as shown in figure 3. The center coordinate of flanging forming square hole is ex in $X$ axis and ey in $Y$ axis. In the first quadrant, according to the geometric characteristics of port, the position of the cross-section is divided into three different regions, which are called latitude crosssectional area, transition area and longitude crosssectional area. Assuming that the angle between the cross 
section and the $X$ axis is $\mathrm{Y}$, the length of segment $\overline{A C}$ can be calculated could be known, as well as in other quadrants, the length of segment $\overline{A C}$ can be calculated, as shown in figue 2 .

Let us assume that

$$
\begin{aligned}
& \gamma_{1}=\arctan \frac{W / 2+e_{y}-R_{3}}{L / 2+e_{x}} \\
& \gamma_{2}=\arctan \frac{W / 2+e_{y}}{L / 2+e_{x}-R_{3}}
\end{aligned}
$$

When $\gamma<\gamma_{1}$

$$
\overline{A C}=\frac{L / 2+e_{x}}{\cos \gamma}
$$

$$
\begin{aligned}
& \text { When } \gamma_{1} \leq \gamma<\gamma_{2} \\
& \overline{A C}=O_{x} \cos \gamma \\
& O_{x}=L / 2+e_{x}-R_{3}+\tan \gamma\left(W / 2+e_{y}-R_{3}\right) \\
& +\sqrt{\left(1+\tan ^{2} \gamma\right) R_{3}^{2}-\left(\tan \gamma\left(L / 2+e_{x}-R_{3}\right)-\left(W / 2+e_{y}-R_{3}\right)\right)}
\end{aligned}
$$

When $\gamma \geq \gamma_{2}$

$$
\overline{A C}=\frac{W / 2+e_{y}}{\sin \gamma}
$$

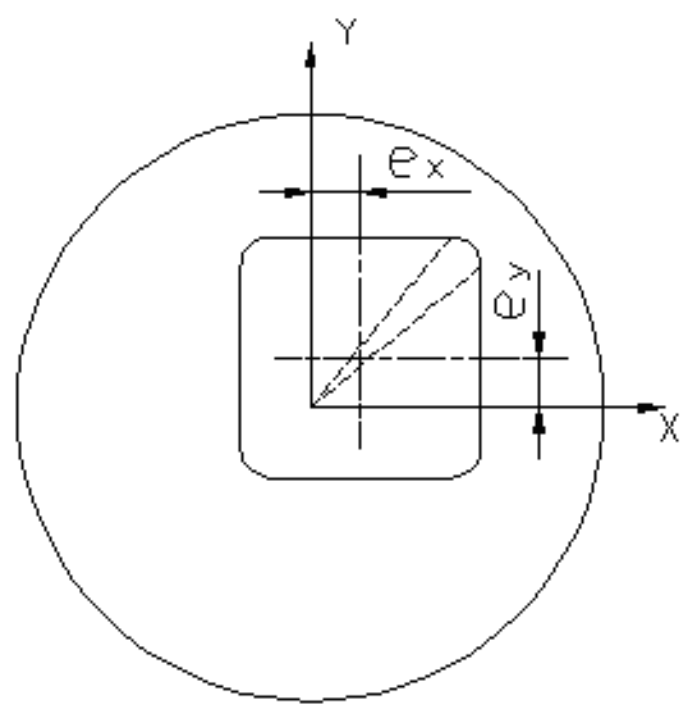

Figue 2. View of flanging forming part in $X O Y$ plane

\section{Solution of Optimized Target Function}

The target function of optimized model built up in this paper is the mean square difference between practical flanged height and ideal height, and it is related to the shape and sizes of the flanged part. Because of the complex flanging process, it is very difficult to find out the values with conventional method, for this reason FEmethod is used to simulate the forming process, and using the results file of the simulation to find out the shape and sizes of the formed parts, further to find out the solution to target function, mean time to judge the constraints.

Taking a forming process as an example, in which the process parameters are as follows, the shell diameter is
$120 \mathrm{~mm}$, width and length of hole is $60 \mathrm{~mm}$, square corner is $5 \mathrm{~mm}$, flange fillet is $5 \mathrm{~mm}$, height of flanging is $55 \mathrm{~mm}$ and blank thickness is $2 \mathrm{~mm}$,the FE model is built. Through the simulation calculation, we can get all kinds of deformation parameters of the workpiece graphics, in which forming limit diagram as shown in Figure 3. In the optimization model, the objective function is related to by the height of the flanging port, which can be read out from the results of simulation calculation through independent development of the program.

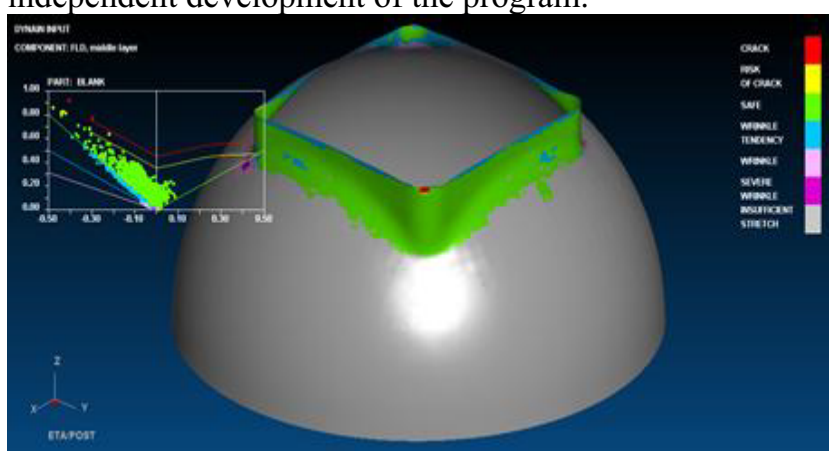

Figue 3. Results of finite element simulation

\section{Optimized Design}

According to the principle of length, linear material size of before and after flanging deformation is unchanged, so in the same direction, the flanging height changes is equal to the length change of radial length. Size difference of prefabricated hole and ideal prefabricated which can be calculated ideal prefabricated hole shape can be obtained through calculation difference of the flanging height and ideal flanging height. In order to obtain more accurate size of prefabricated hole, iterative method is applied in this paper.

Through the optimization calculation of flanging forming,we obtain a workpiece such as shown in Figure 4. From the figure we can see port flanging is very smooth and there isn't any wrinkle and crack meeting the first constraint conditions of the optimization model. The average height of flanging forming is $55.01 \mathrm{~mm}$, the mean square difference is 0.004 , the maximum value is $55.16 \mathrm{~mm}$, the minimum value is $54.78 \mathrm{~mm}$, maximum difference of height is $0.22 \mathrm{~mm}$ meeting the second constraint conditions of the optimization model.

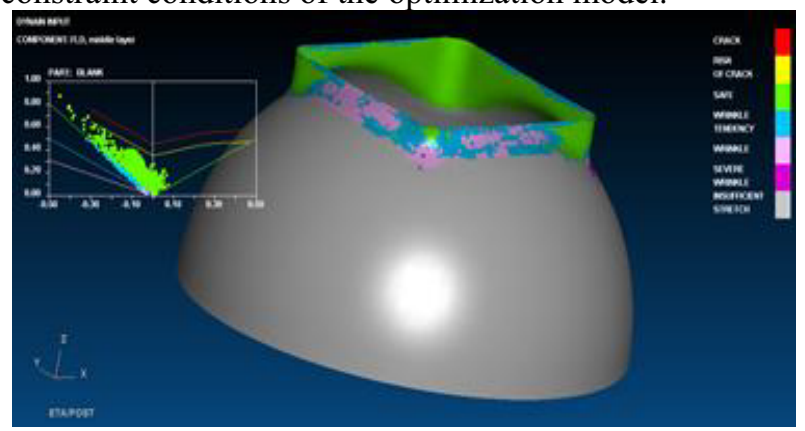

Figue 4. Results of optimization analysis

\section{Summary}

Study on the optimization design of the eccentric hole flanging forming prefabricated hole shape and size. We 
establish shell flanging forming prefabricated hole optimization design model, to solve the mathematical model value as the optimization design of initial value, to a limited simulation results as the objective function solution solver, on the shell side hole flanging forming prefabricated hole shape and size optimization design. Optimized design examples show that through two times of optimization design calculation, the mean square error is 0.004 , the maximum height difference is $0.22 \mathrm{~mm}$, the optimal design is fast, and the design results meet the requirements of the process.

\section{Acknowledgements}

This work was supported by the Doctoral Foundation of Shandong Jianzhu University (XNBS1245).

\section{References}

[1] Y.T Luo. Rocket Propulsion,2,50(2009).

[2] J.S Liu, B Wang,J.H Tian. Plasticity Engineering. 12,74(2005).

[3]B Wang,J.S Liu. Journal of Taiyuan University of Technology. 3,161(2005).

[4]Y.P Zhang,B Wang,J.S Liu. Journal of Taiyuan University of Technology. 4, 437 (2006).

[5]S.L Heng,C.Y Lee,C.H Wub. Machine Tools \& Manufacture, 47,168(2007).

[6]L Gu, J Zhang, L Zhu.Forging and Stamping Technology,3,71(2009). 